\title{
The impact of different Milling Machines on Marginal Fit Discrepancies of Lithium Disilicate Crowns
}

\author{
FLORIN I. TOPALA ${ }^{1}$, MIRCEA RIVIS ${ }^{2 *}$, SIMONA I. HATEGAN ${ }^{*}$, ANDREEA SECOSAN ${ }^{1}$, \\ RAUL ROTAR ${ }^{1}$, EMANUEL BRATU ${ }^{3}$, ANCA JIVANESCU ${ }^{1}$ \\ ${ }^{1}$ University of Medicine and Pharmacy "Victor Babes" from Timisoara, Faculty of Dental Medicine, Department of \\ Prosthodontics, 9 Revolutiei din 1989 Blvd., 300041, Timisoara, Romania \\ ${ }^{2}$ University of Medicine and Pharmacy "Victor Babes" from Timisoara, Faculty of Dental Medicine, Department of Oral \\ Surgery and Anesthesiology , 9 Revolutiei din 1989 Blvd., 300041, Timisoara, Romania \\ ${ }^{3}$ University of Medicine and Pharmacy "Victor Babes" from Timisoara, Faculty of Dental Medicine, Department of \\ Implant Supported Restorations 9 Revolutiei din 1989 Blvd., 300041, Timisoara, Romania
}

Abstract. The goal of this study was to determine the level of various discrepancies in all-ceramic IPS e. max - lithium disilicated crowns milled with three different milling machines. A $3 D$ printed Geller model with a preparation for all ceramic crown was scanned, then the digital design was created and 24 lithium disilicate crowns were milled using three different milling machines: Plan mill 40S (Planmeca, Helsinki, Finland), Cerec MC XL (Sirona GmbH, Germany), and one laboratory milling machine Imes-Icore 650i (Coritec, Eiterfeld, Germany). Among the three groups, the highest marginal fit accuracy was displayed by crowns fabricated by laboratory milling machine Imes-Icore 650i, followed by chairside Plan mill 40S and Cerec MC XL. The results of our study shows that llithium disilicate crowns fabricated by a laboratory milling and 2 chair-side milling machines present marginal accuracy in the acceptable clinical range.

Keywords: 3D printed Geller model, CAD/CAM systems, milling machines, marginal fit

\section{Introduction}

Increasing patient's and clinicians demand for esthetics in the last decade, has led to the development of new technologies and ceramic materials. CAD/CAM systems evolved constantly and create the possibility to fabricate prosthetic restorations with an enhanced accuracy of marginal fit [1].

An effective digital dental treatment plan involves shorter sessions, with higher comfort for the patient, by replacing the conventional impression technique with the digital impression. Chairside CAD/CAM systems, can diminish the working time for both the dentist and the patient by decreasing the need for the dental laboratory phase and creating the possibility of same day restorations.

The crucial footprint of success over time for the fixed dental prostheses is influenced by the marginal trueness of the final restoration. Marginal fit can be defined as the space between the boundary of the prepared tooth and the edge of the prosthetic work. This prevents the development of secondary caries, accumulation of bacterial plaque, infiltration of bacteria and toxin [2]. All of these factors can generate periodontal inflammatory processes. Of paramount importance is the tooth preparation type because, in default of a proper preparation and a ferrule effect, we can't have an optimal marginal adaptation.

Due to the technological breakthroughs and the benefits of CAD/CAM systems, several wellknown companies have improved both the quality of dental digital impressions and shorten the time of data acquisition and milling process.

There are currently CAM systems that gather up four or five milling axes. One study focused on these topics has shown that the five-axis milling machines are defined by a special precision $[3,4)]$.

Another study has shown that the five-axis CAM component is highly superior in terms of accuracy to those with four axes; moreover, a paramount importance is also given to the diameter of the cutter: the smaller the diameter is, the more accurate the prosthetic part is [5].

\footnotetext{
*email:mrivis@yahoo.com, hategan.simona@umft.ro
} 
The most utilized materials produced with CAD/CAM technology are zirconia and lithium disilicate. From the point of view of marginal adaptation, one study has shown that there are no significant differences between pressed and milling process of fabrication for lithium disilicate crowns [1]. Another study evaluates the marginal fit of milled lithium disilicate crowns with modern $2 \mathrm{D}$ and 3D micro CT analysis [6].

Some authors had shown that zirconium dioxide crowns that were manufactured with a chairside CAD/CAM system displayed significantly smaller marginal gaps than those made by scanning dental casts [7].

Another study has shown that even though CAD-CAM zirconia copings presented the best marginal fit among the experimental groups, no significant differences were noticed between CADCAM zirconia and lithium disilicate crowns [8].

The purpose of this study is to compare the marginal fit of lithium disilicate crowns milled with two chairside Planmill 40S (Planmeca, Helsinki, Finland), Cerec MC XL (Sirona GmbH, Germany), and one laboratory milling machine Imes-Icore 650i (Coritec, Eiterfeld, Germany). Since the precision of marginal fit is crucial for durability in time for all ceramic restorations and for the best results in dental treatments.

\section{Materials and methods}

A central incisor, (\#2.2.) on a 26-year-old patient, was prepared for an all-ceramic crown with a chamfer finish line. In addition, a conventional impression with polyether (Impregum Penta, 3M ESPE) was taken in order to obtain a gypsum model, that was subsequently scanned to achieve a 3D model.

Using a 3D printer (Formlabs-2, Berlin, Germany), the STL file was processed in order to print a Geller model with removable dies and teeth (Figure 1).

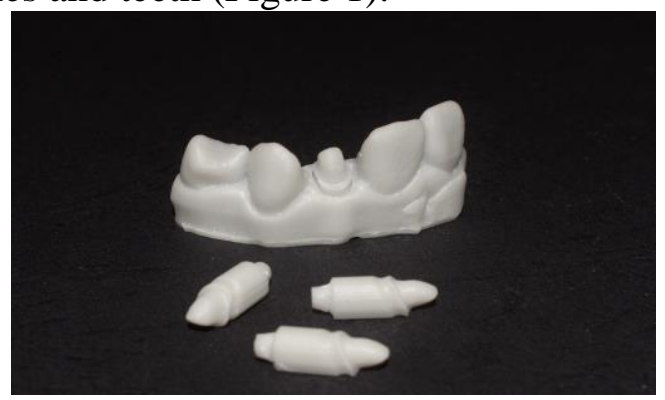

Figure 1. 3 D printed Geller model

In pursuance of simulating the same \#2.2 tooth abutment, the removable dies were printed three times. After each print, the Geller model was scanned with three different scanners: Planscan ( Planmeca, Helsinki, Finland), CEREC Omnicam (Sirona GmbH, Germany), and one laboratory scanner 3Shape D2000 (Copenhagen, Denmark). The digital model was generated, than the digital design of an all ceramic crown was created using two soft-wares: PlanCAD easy (Planmeca, Helsinki, Finland) (Figure 2), CEREC Omnicam (Sirona GmbH, Germany) .

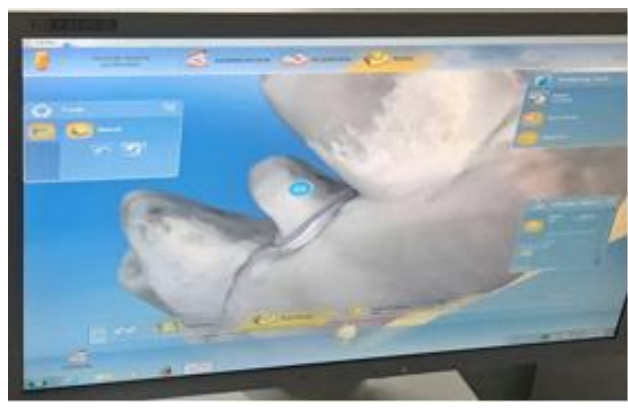

Figure 2. Digital design with Cerec 


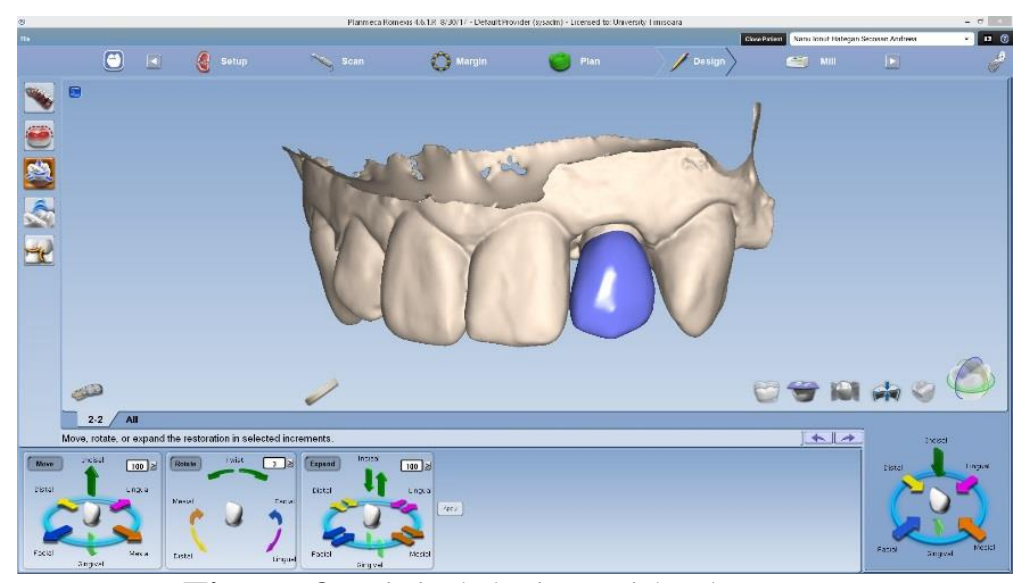

Figure 3. Digital design with Planmeca

For each system, 8 IPS e. max CAD - lithium disilicate all ceramic crowns were milled using three different milling machines: two chairside Planmill 40S (Planmeca, Helsinki, Finland), Cerec MC XL (Sirona GmbH, Germany), and one laboratory milling machine Imes-Icore 650i (Coritec, Eiterfeld, Germany) (Figure 4).

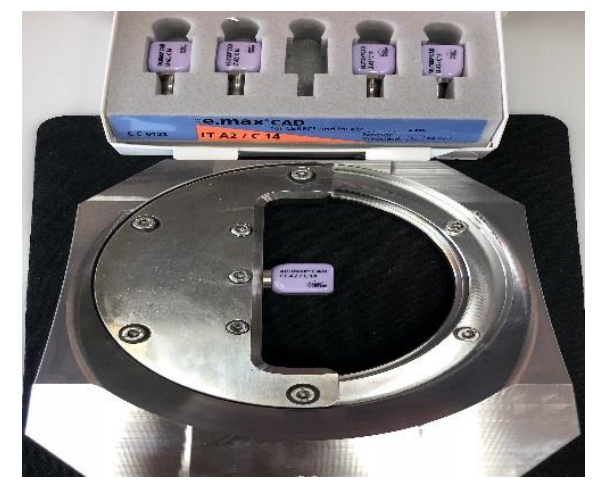

Figure 4. Milling machine-Coritec 650i

(imes-icore, Germany)

The milled all-ceramic crowns and printed abutments were attached to an appropriate printed plastic fixation stand (Figure 5).

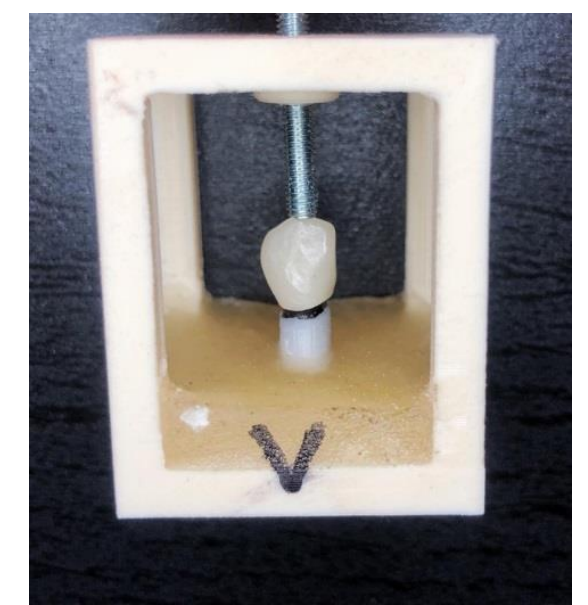

Figure 5 Milled all-ceramic crown in printed plastic fixation stand 
Two holes were drilled in the stand, one on the base and one on the top, corresponding to the dimensions of the mini-screws which hold and allow the examination of the interface between the crown and the printed abutment. In order to determine the accuracy of marginal fit, the margins of ceramic crowns were outlined with a circumferential $1 \mathrm{~mm}$ black track line. The same step was repeated on the abutment where the axial wall adjacent to the chamfer finish line served as a contrast in order to determine the differences between the marginal gap and the crown margin. A calibration grid was used for each, 100 microns apart, in order to record the same focus as those of the specimen's photographs (Figure 6).

Each axial surface (mesial, distal, buccal and oral) were separately analyzed by photographing at 40x magnification with a digital camera (D3300, Nikkon) mounted on a stereomicroscope (Edmund EZoom) (Figure 7,8).

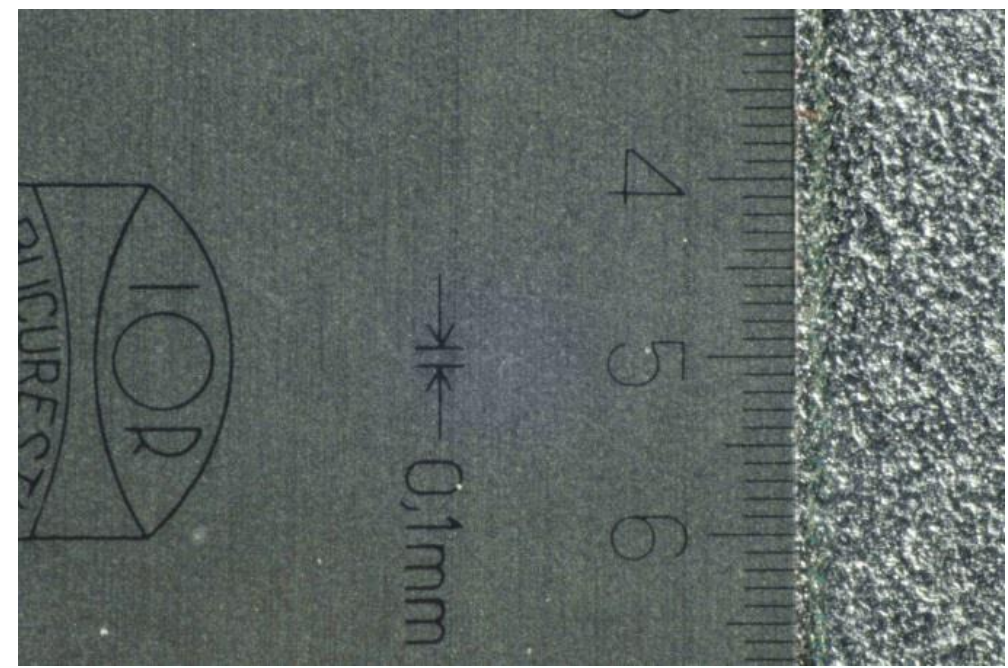

Figure 6. Calibration grid

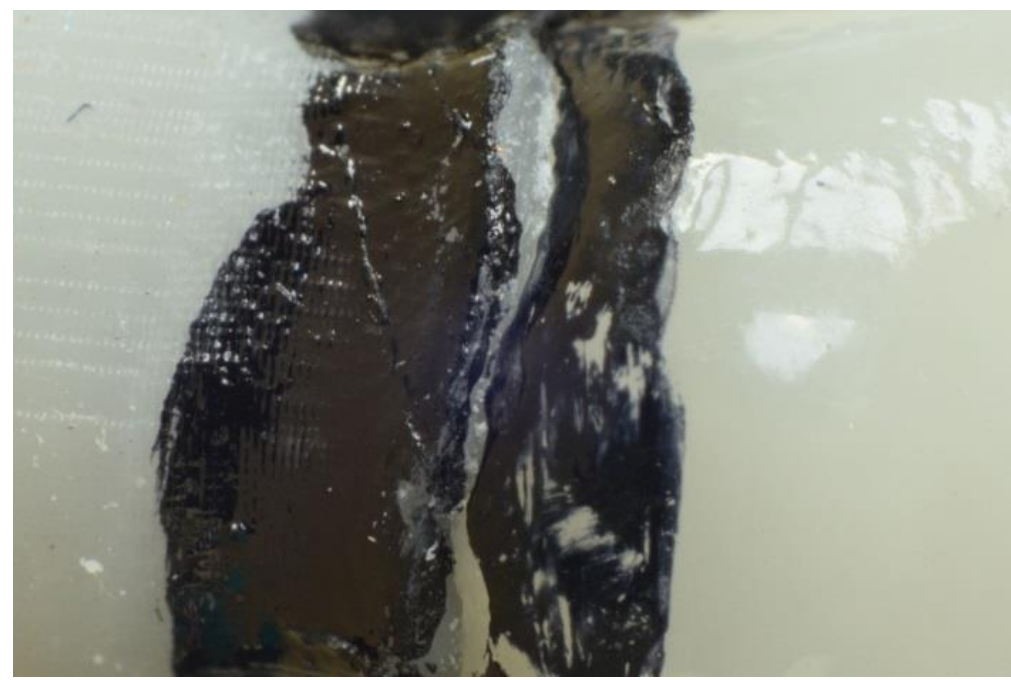

Figure 7. Microscopic image of Cerec e.max crown marginal fit 


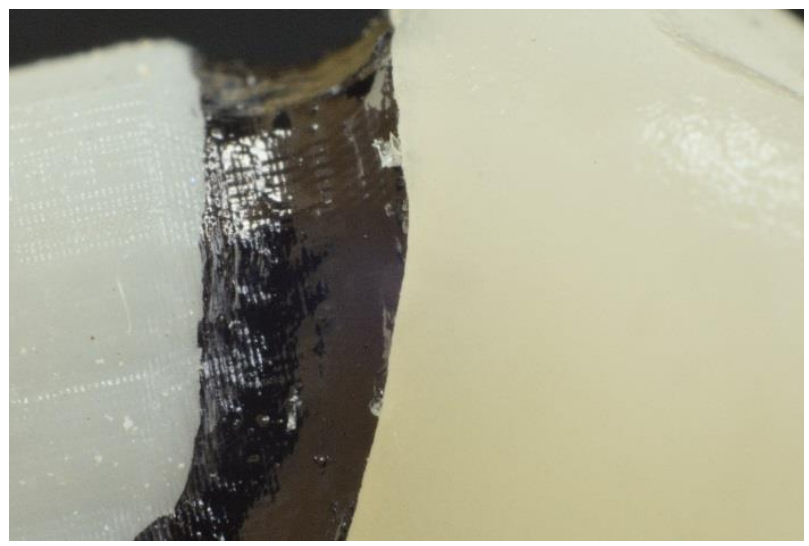

Figure 8. Microscopic image of Planmeca e.max crown marginal fit

For each surface (buccal, palatal, mesial and distal), four vertical crown margin to apex measurements were determined and an average was noted in the table. The marginal gap of the specimen was measured using the software Image J, a Java-based image processing program (U.S. National Institutes of Health) by defining the known distance on the measuring scale picture in micrometers in Pixel units and, thereby, obtained pixel/micrometer relationship could be globally applied for all pictures. The measurements are presented in results and discussion section.

Statistical analysis was preformed using the Kolmogorov-Smirnov test of normality to assess data distribution. Statistical significance was assessed using One Way ANOVA test.

\section{Results and discussions}

Mean values of the marginal gap were calculated including all the surfaces analyzed (buccal, palatal, mesial and distal), for all the individual samples. The results presented statistical significance with a $p$ value $<0.01$. In all three groups, the mesial surface presented the highest marginal gap values (Table 1).

Table 1. Marginal gap on each investigated surface $(\mu \mathrm{m})$

\begin{tabular}{|cccccc|}
\hline & & $\mathrm{V}$ & $\mathrm{M}$ & $\mathrm{O}$ & $\mathrm{D}$ \\
\hline CEREC Omnicam & 1 & 166.8 & 175.9 & 124.6 & 77.6 \\
\hline & 2 & 143.6 & 162.2 & 122.3 & 70.9 \\
\hline & 3 & 150.1 & 166.4 & 124.6 & 74.1 \\
\hline & 4 & 147.3 & 170.9 & 117.9 & 68.7 \\
\hline & 5 & 161.0 & 173.6 & 119.3 & 74.9 \\
\hline 3 shape & 1 & 61.4 & 88.9 & 55.0 & 54.5 \\
\hline & 2 & 58.3 & 81.1 & 56.3 & 55.7 \\
\hline & 3 & 62.0 & 86.9 & 52.7 & 53.2 \\
\hline & 4 & 58.7 & 84.9 & 54.5 & 55.0 \\
\hline PLANMECA Planscan & 5 & 61.5 & 87.3 & 50.9 & 52.1 \\
\hline & 1 & 95.5 & 102.3 & 47.7 & 95.7 \\
\hline & 2 & 97.2 & 95.8 & 50.5 & 100.6 \\
\hline & 3 & 94.5 & 99.3 & 48.7 & 99.4 \\
\hline & 4 & 89.7 & 103.7 & 44.9 & 92.8 \\
\hline & 5 & 93.3 & 100.3 & 47.4 & 96.6 \\
\hline
\end{tabular}

The results showed that the best marginal fit was presented in the "3Shape D2000 group" $(62.9 \pm 12.2 \mu \mathrm{m})$, followed by "Planmeca “(82.8 $\pm 25.9 \mu \mathrm{m})$ and "CEREC “(128.8 $\pm 40.3 \mu \mathrm{m})$ groups'. (Figure 8) 


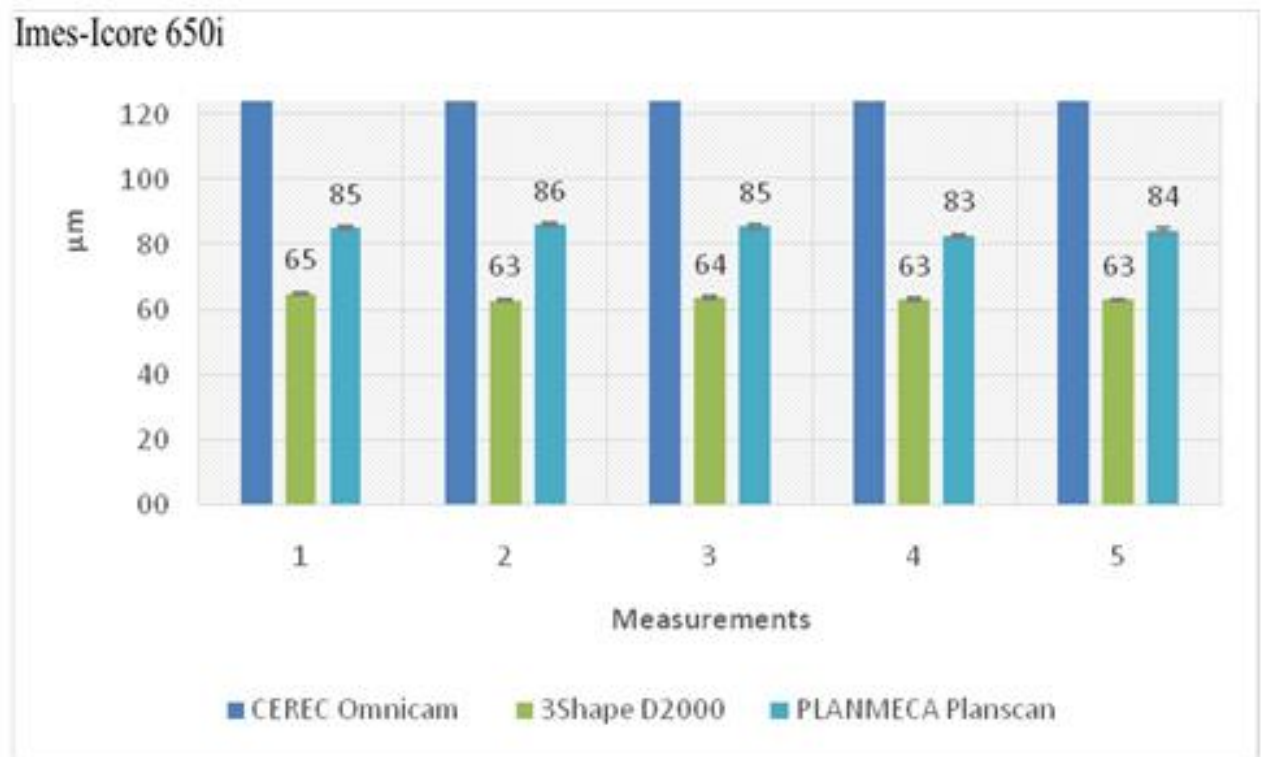

Figure 8. Mean values of the marginal gap

Perfect marginal fit is the goal of every restoration, in order to obtain longevity and success. Recent studies have shown that a marginal adaptation within the range of $50-120 \mu \mathrm{m}$ is acceptable from the clinical perspective. [2-12]

Several researchers reviewed various milling approaches regarding the precision of the final restoration. One study has shown that five-axis milling machines are much more reliable than the fouraxis milling machines. Considering the marginal adaptation, the five-axis CAM component such as IMES CORITEC $(33.9 \pm 16.3 \mu \mathrm{m})$, in LAB MCX5 $(32.3 \pm 9.7 \mu \mathrm{m})$ shows a much better marginal fit than the components with four axes, such as CEREC MCXL $(62.1 \pm 17.1 \mu \mathrm{m})$ [3]. A different study has been conducted and the findings were similar, i.e., the five-axis milling machines are more reliable $(41+/-15 \mu \mathrm{m})$ than those with four axes (from 61 to $96 \mu \mathrm{m})$ [4].

A comparison study made between the conventional and the milling method used in order to obtain a final restoration from lithium disilicate showed that, in terms of the marginal adaptation, the technique utilized has values between 90 and $130 \mu \mathrm{m}$ while the CAD/CAM system has values between 87 and $115 \mu \mathrm{m}$. Furthermore, in some studies, the Anova test showed that there is no significant difference in the manufacturing methods in terms of the marginal adaptation [5].

Other similar studies showed that the CAD/CAM system has lower values in terms of the marginal adaptation (i.e. approx. $48+/-25 \mu \mathrm{m}$ ) compared to the conventional techniques whose values are within the $74+/-47 \mu \mathrm{m}$ range [7].

Numerous studies have been conducted to see the precision and accuracy of different CAD/CAM system. There are studies that conclude that the pressed ceramic technique has higher values than those that use the CAD/CAM systems in terms of the marginal adaptation, however, all values are below 120 $\mu \mathrm{m}[8]$.

One study outlined the difference in marginal adaptation between the CEREC CAD/CAM system and the TRIOS CAD combined with Wieland Zenotec CAM system. The values for the CEREC system were ranging between 111.07 (+/-6.33) $\mu \mathrm{m}$ while those for Trios were somewhere to $60.17(+/$ 11.09) $\mu \mathrm{m}$. The post-hoc Anova and Scheffe tests showed statistically significant differences in the marginal adaptation between the two systems [9].

Another study assessed the difference between five CAD/CAM systems, dividing them into five different groups: Group A is composed of Ceramill-Motion 2 systems, AmannGirrbach; Group B gathers the Wieland, IvoclarVivadent systems; Group C involves the Cerec, IvoclarVivadent systems; Group D gathers up the Prettau Zirconia, Zirkonzahn systems, and Group E, the Cad4dent system. The lowest marginal adaptation values were $46.93+/-13.50 \mu \mathrm{m}$ and the highest values, i.e. $101.65+/$ - 
$35.56 \mu \mathrm{m}$, were found in groups A and D. The Anova test showed a statistically significant difference across all groups. Using the post hoc test, Turkey HSD test showed statistically significant differences between Group A and all other groups except for B; B with D; C with A; D with A; B and E with A. Definitely, the marginal adaptation to CAD/CAM systems is highly influenced by the technique of making prosthetic parts as well as by the considerable variations existing in different systems [10-12].

Marginal adaptation is also influenced by the finish-line of preparation. Depending on the finishline design, there are several studies. It can be noticed that at a certain preparation angle, the marginal fracture of the restoration may occur, but this could only be noticed at a 60 -degree angle. The mean of the marginal fractures in CEREC system was $2.8 \%$ at right angles $\left(90^{\circ}\right) ; 3.5 \%$ at 30 degree and $10 \%$ at 60-degree angles, respectively [13-17].

Consequently, the marginal adaptation of a prosthetic restoration plays an essential role in terms of the biological, the mechanical and the aesthetic factors and directly influece the longevity and clinical success of the restoration [18], [19]. All this studies lead to the similar results with our study that the pressed ceramic technique has highest values.

\section{Conclusions}

With all the limitation of this study, it can be concluded that despite the fact that lithium disilicate crowns fabricated with a laboratory milling machine had shown a higher precision in marginal fit, all crowns milled with chairside milling machines present marginal adaptation in an acceptable clinical range.

\section{References}

1.DOLEV E, BITTERMAN Y, MEIROWITZ MA. Comparison of marginal fir between CAD/CAM and hot press lithium disilicate crowns. J Prosthet dent; 121; 1.2019:124-128.

2.ROSENSTEIN SF, LAND MF, FUJIMOTO. Contemporary fixed prosthodontics. St. Louis, Mo: Mosby Elsevier. 2016

3.ROBERTO R, ASSR H, SOARS-PORTO T, LANG L, TEACH S. Are different generations of CAD/CAM milling machines capable to produce restorations with similar quality?, J Clin Exp Dent. 2016. 8(4): e423-8.

4.KIRSCH C, ENDER A, ATTIN T, MEHL A. Truness of four different milling procedures used in dental CAD/CAM systems; Clin Oral Investig. 2017.21(2):551-558.

5.BOSCH G, ENDER A, MEHL A. A 3-dimensional accuracy analysis of chairside CAD/CAM milling processes; J Prosthet Dent. 2014.112:1425-1431.

6.DE FREITAS BN, BRUNA N, TONIN BSH, MACEDO AP, \&all. Adaptation accuracy of milled lithium disilicate crowns: A 2D and 3D micro CT analysis. J Est Rest Dent, epub 2020; doi:10.1111/jerd.12574.

7.EUAN R, FIGUERAS-ALVAREZ O, CABRATOSA-TERMES J, OLIVER- PARRA R. Marginal adaptation of zirconium dioxide copings: influence of the CAD/CAM system and the finish line design. J Prosthet Dent. 2014.12:155e62.

8.RICCITIELLO F, AMATO M, LEONE R., SPAGNUOLO G., SORRENTINO R. In vitro Evaluation of the Marginal Fit and Internal Adaptation of Zirconia and Lithium Disilicate Single Crowns: Micro-CT Comparison Between Different Manufacturing Procedures. The Open Dentistry Journal. 2018. 12. 160-172.

9.TRIC O. "The Carrot Model" Spectrum dialogue. 2010.Vol. 9 No. 2

10.NG J, RUSE D, WYATT C., A Comparison of the Marginal Fit of Crowns Fabricated with Digital and Conventional Methods; J Prosthet Dent. 2014. 112(3): 555-60.

11.CANDEA, A., A. JIVANESCU, M. LERETTER \& M. ROMINU. Marginal And Internal Fit Of PartialRetainer $\mathrm{Cad} / \mathrm{Cam}$ Zirconia Fixed Dental Prostheses. Proceedings of the Romanian Academy Series aMathematics Physics Technical Sciences Information Science, 2017;18: 345-352.

12.AZAR B, ECKERT S, KUNKELA J, INGR T, MOUNAJJED R. The Marginal Fit of Lithium Disilicate Crowns: Press Vs. CAD/CAM; Braz. Oral Res. 2018. 32: E00 1

13.ALQAHTANI F. Marginal Fit of All-Ceramic Crowns Fabricated Using Two Extraoral CAD/CAM Systems in Comparison with The Conventional Technique; Clinical, Cosmetic and Investigational Dentistry. 2017.9 1318 
14.HABIB SR, RASHID S; OTAIBI A, KHALES A, ANAZI A, ALI T, MOSLEH S. Comparison Between Five CAD/CAM Systems for Fit of Zirconia Copings. Quintessence International. 2018.Vol. 49 Issue 6, 437444.

15.GIANNETOPOULOS S, VANNOORT R, TSITROU E. Evaluation The Marginal Integrity Of Ceramic Copings With Different Marginal Angles Using Two Different CAD/CAM Systems; J Dent. 2010. 38(12):980616. JIVANESCU A, BIRKENHEIER N, ROTAR R, TOPALA F, GOGUTA L. Marginal Fit of Ceramic Crowns Fabricated with Cad-Cam Technology Using A Direct and Indirect Digital Workflow. Journal of Clinical and Research Medicine. 2019, vol III, Issue 3; 1-6.

17. URAL C, BURGAZ Y, SARAC D. In Vitro Evaluation of Marginal Adaptation in Five Ceramic Restoration Fabricating Techniques. Quintessence Int. 2010.41:585-90.

18.POPESCU, S.M., LASCU, L., GINGU, O., MERCUT, V., OSIAC, E., SCRIECIU, M., TUCULINA, M.J., DRAGHICI, E.C., MANOLEA, H.O., MORARU, A.I., OCT Application in Direct Dental Restorations Marginal Fit Evaluation, Rev. Chim., 70(4), 2019, 1439-1444.

19.NEGUCIOIU, M., POPA, D., CONDOR, D., CUlCITCHI, C., PRODAN, D., MOLDOVAN, M., BUDURU, S., Multivariate Assessment of Marginal Fit Concerning Two Types of Dental Inlays Obtained Through Two CAD-CAM Technology Scanning Methods - an in vitro Follow-up Study, Rev. Chim., 70(9), 2019, 3136-3141.

Manuscript received: 6.04.2020 